DOI: https://doi.org/10.46296/yc.v5i9edespsoct.0116

\title{
LA TECNOLOGIA EDUCATIVA PARA EL APRENDIZAJE DE LA MATEMÁTICA EN LA EDUCACIÓN GENERAL BÁSICA SUPERIOR
}

\section{EDUCATIONAL TECHNOLOGY FOR THE LEARNING OF MATHEMATICS IN GENERAL BASIC HIGHER EDUCATION}

\author{
Vélez-Briones Mónica Melania ${ }^{1}$; De la Peña-Consuegra Geilert ${ }^{2}$ \\ ${ }^{1}$ Licenciada en Contabilidad y Auditoría, Licenciada en Servicios Gerenciales, Docente \\ del Distrito 13D02, Unidad Educativa Camilo Ponce Enríquez. Manta, Ecuador. Correo: \\ lamonikvelezbriones@gmail.com. ORCID ID: https://orcid.org/0000-0002-6682-8244 \\ ${ }^{2}$ Licenciado en Educación, Especialidad Biología, Doctor en Ciencias Pedagógicas, \\ Profesor de la Universidad Técnica de Manabí. Portoviejo, Ecuador. Correo: \\ geilet.delapena@utm.edu.ec. ORCID ID: https://orcid.org/0000-0003-3765-9143
}

\section{Resumen}

En la educación el surgimiento de alternativas en el uso de tecnologías educativas y los soportes metodológicos y didácticos de ejecución son cada vez más regulares en el desarrollo de este campo. Los docentes aún no dominan ni se encuentra lo suficientemente preparado para lograr un proceso innovador, efectivo y actualizado, desde el empleo de las tecnologías de la información y las comunicaciones, lo que incluso repercute en los estudiantes. Se requirió de un estudio a partir de la aplicación de variantes sobre el empleo de las TIC en el proceso de enseñanza aprendizaje de la matemática en el nivel Básico Superior de la Unidad Educativa "Camilo Ponce Enríquez" de Manta. En específico, en el área de la matemática resulta de mayor complejidad, ya que están presentes criterios de mayor abstracción, propio de la naturaleza del conociendo matemático. Se trabajó en un estudio con un enfoque mixto, cuali-cuantitativo, de tipo experimental y transversal. En el cual, se utilizaron una serie de métodos y técnica de naturaleza teórica, empírica y estadística. Los principales resultados logrados se ajustan a la sistematización de criterios y elementos de naturaleza teórica, así como la debida información que responde al proceso de investigación, donde se expresa el nivel de avances en el aprendizaje de los estudiantes de la muestra y la incidencia de los docentes en el proceso que facilitan a partir de la mediación de las tecnologías.

Palabras claves: Tecnología Educativa; Tecnología de la Información y las Comunicaciones; Innovación educativa; Matemática.

\begin{abstract}
In education, the emergence of alternatives in the use of educational technologies and the methodological and didactic supports of execution are increasingly regular in the development of this field. Teachers are not yet proficient or sufficiently prepared to achieve an innovative, effective and updated process, from the use of information and communication technologies, which even affects students. A study was required based on the application of variants on the use of ICT in the teaching-learning process of mathematics at the Superior Basic level of the "Camilo Ponce Enríquez" Educational Unit in Manta. Specifically, in the area of mathematics it is more complex, since there are more abstract criteria, typical of the nature of knowing mathematics. A study was carried out with a mixed, quali-quantitative, experimental and cross-sectional approach. In which, a series of methods and techniques of a theoretical, empirical and statistical nature were used. The main results achieved are adjusted to the systematization of criteria and elements of a theoretical nature, as well as the proper information that responds to the research process, which expresses the level of progress in the learning of the students in the sample and the incidence of the teachers in the process that they facilitate from the mediation of technologies.
\end{abstract}

Keywords: Educational Technology; Information and Communications Technology; Educational innovation; Mathematica.

Información del manuscrito:

Fecha de recepción: 02 de julio de 2021 .

Fecha de aceptación: 01 de octubre de 2021.

Fecha de publicación: 04 de octubre de 2021. 


\section{Introducción}

Hoy por hoy la educación se encuentra inmersa en un proceso de renovación constante debido a la inferencia de dos hechos fundamentales: el surgimiento de nuevas tecnologías y el desarrollo de nuevas metodologías activas que lideran los procesos de enseñanza y aprendizaje en las aulas del nivel de instrucción básica superior.

Dichos procesos se basan en la implementación de la virtualización, del "aprendizaje invertido" y las "TIC" como modelos de enseñanza destinados a mejorar factores como el interés de aprendizaje, motivación y autonomía (Parra, López, Segura, \& Fuentes, 2020). La implementación de ambas metodologías como parte de la estrategia pedagógica provoca una mejora en los procesos de aprendizaje de los estudiantes, en sus logros y en su entusiasmo.

El rápido desarrollo de la tecnología tuvo claramente un impacto significativo en el mundo de la educación. Uno de ellos es con las tecnologías de la información y las comunicaciones (TIC) que se utilizan comúnmente como un recurso de aprendizaje, ya sea en forma de medios de instrucción o simplemente como un depósito de material, e incluso la gestión de actividades de aprendizaje, como por ejemplo el aprendizaje electrónico, aprendizaje a distancia, entre otras. La interacción de los estudiantes con las TIC ciertamente estaba aumentando, debido a las demandas del proceso de aprendizaje (aparte de las razones relacionadas con la propiedad de los dispositivos, la necesidad de autorrealización, etc.). Por tanto, las TIC se vuelven importantes para ser una preocupación para los educadores (Davis, J. P., \& Bellocchi, A, 2020)

Esta condición plantea cuestiones importantes sobre la importancia del dominio de las TIC para los profesores. La integración de las TIC en el aula siempre ha sido una tarea desafiante para muchos profesores. El docente no se siente del todo preparado antes de comenzar a aprender utilizando las TIC, en parte debido al dominio del marco teórico que se relaciona con el desarrollo de las TIC para el aprendizaje de sus estudiantes. 


\section{Metodología}

Para el desarrollo de esta investigación de enfoque mixto, se realizó una revisión a la literatura relacionada con el uso de las TIC y la enseñanza de las matemáticas. Consecuentemente se realizó un cuasi-experimento que se expresa en la necesidad de conocer lo relativo al desarrollo de actividades en el proceso de enseñanza aprendizaje de la matemática con empleo de una muestra total de 63 estudiantes de la educación básica superior de la unidad educativa "Camilo Ponce Enríquez". Para lo cual se distribuyeron en un grupo de control con 31 estudiantes y el grupo experimental con un total de 32 estudiantes correspondiente a los paralelos A y B de educación básica.

El cuasi-experimento se utilizó en función de determinar la viabilidad de las TIC como estrategia metodológica de enseñanza y aprendizaje de las matemáticas en los estudiantes de educación básica superior de la unidad educativa "Camilo Ponce Enríquez".

\section{Resultados}

El sistema educativo, es responsable de la formación de cada nueva generación, y consecuentemente se adapta a sí mismo a lo largo de los años a los cambios en la sociedad circundante. Los cambios tecnológicos, la revolución de la información y los cambios en los hábitos de trabajo requieren un cambio fundamental en los métodos de enseñanza, de modo que pueda preparar adecuadamente a las generaciones futuras para la realidad moderna (Naifeld, Edni \& Simon, Eitan, 2017).

La capacidad de pensar críticamente se considera uno de los resultados deseables de una educación de primera línea. El desarrollo del pensamiento crítico en los estudiantes contemporáneos permite a los futuros especialistas dominar con éxito las bases de su futura profesión y estar preparados para vivir y trabajar de manera creativa en el siglo XXI, dado que preparar a los estudiantes universitarios para el futuro en la clave de cualquier educación.

Bajo esta premisa, los docentes deben tener en cuenta que el éxito 
de un líder potencial va más allá del conocimiento teórico. Por ello, resulta de suma importancia enfatizar en los resultados del aprendizaje autodirigidos y orientados a la propiedad, puesto que se espera que los profesionales asuman la responsabilidad por sus decisiones.

A los jóvenes estudiantes de la educación básica superior les resulta difícil conceptualizar estos conceptos y describirlos de manera superficial y concreta. Se necesita mediación para conectar el aprendizaje matemático de estos conceptos con el trabajo práctico de los estudiantes desde un contexto empírico.

El estudiante es un actor clave en el proceso de aprendizaje. Los estudiantes contemporáneos son conocedores de la tecnología, prefieren el aprendizaje práctico y experiencial, desean resultados significativos e interactivos, Por lo tanto, es importante comprender sus expectativas educativas (Utomo, 2015; Warford, 2017; Velázquez, 2021). Como educador, es importante para enseñarles de una manera que no solo cumpla con sus expectativas, sino que también vaya más allá de eso para satisfacerlos y comprometerlos.

Para superar esta lucha, el papel del docente es lo más importante. La "presencia docente" del instructor desempeña una función papel más relevante en la provisión de diseño, facilitación e instrucción directa. En el aprendizaje de orden superior, la interacción y la discusión juegan un papel importante, pero deben estar respaldados por el diseño y el liderazgo del instructor (Silva, 2018; Siakas Et al., 2019; Singh, 2020).

El entorno de innovación se modela a través de un centro de formación y práctica centrado en el alumno, que realiza actividades educativas basadas en un enfoque de competencias. La base conceptual de la tecnología son los principios rectores de la pedagogía en asociación, la educación innovadora, la competencia y los métodos interdisciplinarios y la tecnología de enseñanza centrada en el estudiante. La parte de contenido técnico se basa en los estándares educativos actuales para colegios y universidades, modelando la educación en ciencias naturales y matemáticas. El componente de procedimiento de la tecnología es 
una serie de formas y métodos innovadores de actividades educativas, que se integran en proyectos de ciencias sociales a largo plazo.

Mediante el uso de la tecnología docente en el proceso educativo, la aplicación de tecnología innovadora en la educación se maneja en el proceso de aprendizaje. Los cursos, narrativas y planes de lecciones, manuales de métodos y el desarrollo de métodos de enseñanza deben combinarse con la descripción del experimento para la experimentación (Sousa Et al., 2015, Stachová, 2019; Tarando; 2021). Además, la tecnología de la enseñanza debe establecer una base metodológica, mecanismos, métodos y herramientas claros, para diagnosticar el proceso educativo y desarrollar las teorías implementadas, y guiarse por los métodos de enseñanza. (Fahriddinqizi, Ashurova, 2020).

Las TIC proporcionan recursos didácticos proporcionando recursos didácticos y utilizando artefactos de incentivo (incluidas herramientas de comunicación y máquinas para procesar información) para proporcionar recursos didácticos, contribuyendo así a la mejora del entorno educativo. Asimismo, los jóvenes deben adquirir los conocimientos y habilidades necesarios para utilizar las TIC para nuevos puestos de trabajo y necesidades académicas para que puedan mejorar la efectividad (optimización de recursos) y eficiencia (haciendo buenas acciones) de la gestión del sistema educativo. (García-Alcaraz Et al., 2018)

Actualmente, las TIC son consideradas como una herramienta para prácticas de enseñanza innovadoras y ofrecen la posibilidad de incluir nuevas estrategias de enseñanza que estimulen el interés y la motivación de los estudiantes para mejorar el proceso de enseñanza dentro y fuera del aula (ChigozieOkwum, 2016).

La capacidad digital sigue siendo un desafío para la práctica docente y la innovación educativa, y requiere una reflexión sobre la integración de las tecnologías de la información y la comunicación en la experiencia docente. 
El complejo proceso de adquisición de capacidades digitales conecta grandes dificultades docentes con la gestión de la información, el uso de métodos de enseñanza TIC, la evaluación de actividades digitales y la colaboración en un entorno virtual (Tippa \& Mane, 2018; Tso \& Lau, 2018; Terziev, 2020).

La imagen de aislamiento en el desarrollo profesional dificulta el intercambio de significados $y$ experiencias y el reconocimiento de los beneficios y la confianza del uso de las TIC. Los profesores deben realizar una formación más coherente para sus necesidades de enseñanza y hacer que el trabajo sistemático de acuerdo con el proyecto sea una buena práctica. Concluyó que es necesario cerrar la brecha entre las capacidades digitales requeridas y las capacidades digitales realmente adquiridas, y desarrollar experiencias de formación independientes, a largo plazo y colaborativas para apoyar la práctica reflexiva y la innovación educativa. (Rossi Cordero, Barajas Frutos, 2018).

Las TIC y las principales instrumentaciones en el proceso de enseñanza aprendizaje de la matemática se tienen en cuenta desde un amplio horizonte para ello.

En correspondencia se concluye en la aproximación que se hace respecto de la temática Riveros y Castro (2011) las TIC se constituyen como medios de enseñanza y como mediadores en el proceso de enseñanza y de aprendizaje para docentes y estudiantes, lo cual incluso puede tener una incidencia positiva o negativa en dependencia del nivel de desarrollo y de uso de las mismas, y de las posibilidades de los docentes para ello. Por tanto, desde esta perspectiva se determina el desarrollo del proceso didáctico de las matemáticas, así como atender las diferencias individuales incluso con el adecuado empleo de las tecnologías educativas, centradas en TIC.

La implementación de diversas herramientas, como pueden ser recursos multimedias, Podcast, software, etc.; destinados específicamente al desarrollo de los contenidos matemáticos permiten establecer una conexión con la realidad, de tal manera que se pueda aprender matemáticas de manera divertida. En este sentido, existen 
una cantidad considerable de sitios web, portales o plataforma en internet que albergan un sinfín de aplicaciones con carácter educativo, tal como lo señala Cerrillo-i-Martínez (2012) en donde se llega incluso a demostrar una revisión y consulta de una serie de ello. Por otro lado, Pabón, (2014) realizó una revisión de alternativas de esta naturaleza que se basan en una experticia adecuada que tienen resultados visibles y publicados en diversas revistas y sitios sobre tecnología educativa en general. Desde esta perspectiva es necesario tener presente que en actualidad en la web, se pueden consultar una serie de aplicaciones educativas de relevancia dentro del área de las matemáticas, como por ejemplo las que se relacionan en la siguiente tabla.

Tabla 1. Aplicaciones y recursos de software para matemáticas en la web.

\begin{tabular}{|c|c|c|}
\hline Categoría & Aplicación & Descripción \\
\hline \multirow[t]{3}{*}{ Aritmética } & $\begin{array}{l}\text { Math Cilenia (en } \\
\text { inglés) }\end{array}$ & $\begin{array}{l}\text { Minijuegos para practicar las operaciones básicas, } \\
\text { destinada a alumnos de primaria }\end{array}$ \\
\hline & $\begin{array}{l}\text { Calculadoras } \\
\text { matemáticas }\end{array}$ & $\begin{array}{l}\text { Selección de diferentes tipos de calculadoras online para } \\
\text { hacer operaciones de forma rápida y sencilla. }\end{array}$ \\
\hline & Ábaco online & $\begin{array}{l}\text { Para representar diferentes números, aprender a sumar } \\
\text { de manera gráfica y trabajar las cifras de otra forma. }\end{array}$ \\
\hline \multirow[t]{2}{*}{ Geometría } & Descartes & $\begin{array}{l}\text { Permite crear objetos interactivos, diseñada } \\
\text { especialmente para la matemática, aunque aplicable } \\
\text { también a otros temas y asignaturas; además de trabajar } \\
\text { geometría, se pueden crear gráficos de álgebra, } \\
\text { estadística o funciones }\end{array}$ \\
\hline & Geogebra & $\begin{array}{l}\text { Software matemático multiplataforma para crear } \\
\text { simulaciones que relacionan el álgebra con la geometría, } \\
\text { para ayudar a los alumnos a comprender los conceptos } \\
\text { de forma visual e interactiva }\end{array}$ \\
\hline \multirow[t]{2}{*}{ Álgebra } & Math Papa & $\begin{array}{l}\text { Calculadora de álgebra que resuelve la ecuación paso a } \\
\text { paso, para que el alumno comprenda el proceso; } \\
\text { también incluye lecciones para aprender o repasar } \\
\text { actividades } \quad \text { interactivas } \\
\text { practicar no solo álgebra sino también otros temas }\end{array}$ \\
\hline & Wiris & $\begin{array}{l}\text { Aplicación online que permite construir y resolver todo } \\
\text { tipo de expresiones algebraicas, con una opción más } \\
\text { sencilla para primaria. }\end{array}$ \\
\hline $\begin{array}{l}\text { Funciones y } \\
\text { gráficas }\end{array}$ & Desmos & $\begin{array}{l}\text { Aplicación online para representar y estudiar funciones } \\
\text { de forma gráfica. Cuenta con una base de datos de } \\
\text { actividades ya creadas por profesores que se puede } \\
\text { utilizar. }\end{array}$ \\
\hline
\end{tabular}




\begin{tabular}{|c|c|c|}
\hline & $\begin{array}{l}\text { Algeo Graphing } \\
\text { Calculator }\end{array}$ & $\begin{array}{l}\text { Aplicación para Android donde se pueden introducir y } \\
\text { dibujar funciones de forma sencilla desde el móvil o la } \\
\text { tableta }\end{array}$ \\
\hline \multirow[t]{2}{*}{ Videos } & Math TV & $\begin{array}{l}\text { Videos a modo de lecciones explicativas sobre diversos } \\
\text { temas de la asignatura, disponibles en inglés, en muchos } \\
\text { casos también en español. }\end{array}$ \\
\hline & Khan Academy & $\begin{array}{l}\text { Lecciones de Matemática organizadas por niveles } \\
\text { educativos y temas para ir aprendiendo poco a poco, } \\
\text { desde lo más básico hasta lo más completo. }\end{array}$ \\
\hline \multirow[t]{4}{*}{$\begin{array}{l}\text { Juegos } \quad y \\
\text { actividades } \\
\text { interactivas }\end{array}$} & $\begin{array}{l}\text { Buzzmath } \quad(e n \\
\text { inglés) }\end{array}$ & $\begin{array}{l}\text { Plataforma online creada por un equipo de profesores de } \\
\text { matemática que cuenta con más de } 3.000 \text { problemas } \\
\text { matemáticos y facilita la enseñanza y el aprendizaje a } \\
\text { través de ejercicios interactivos y visuales }\end{array}$ \\
\hline & Math Game Time & $\begin{array}{l}\text { Repositorio de juegos de matemática de todo tipo, } \\
\text { organizados por niveles o por temas. }\end{array}$ \\
\hline & $\begin{array}{l}\text { Materiales } \\
\text { didácticos del } \\
\text { Proyecto Gauss } \\
\text { para secundaria } \\
\text { y primaria }\end{array}$ & $\begin{array}{l}\text { Recopilación de propuestas interactivas en Java para } \\
\text { practicar } \\
\text { todo tipo de conceptos matemáticos. }\end{array}$ \\
\hline & Amo las mates & $\begin{array}{l}\text { Completa página web con recursos, juegos y material } \\
\text { interactivo para trabajar la matemática en primaria y } \\
\text { secundaria, organizados por niveles y temas. }\end{array}$ \\
\hline \multirow[t]{3}{*}{$\begin{array}{l}\text { Matemática } \\
\text { práctica }\end{array}$} & $\begin{array}{l}\text { Sector } \\
\text { Matemática }\end{array}$ & $\begin{array}{l}\text { Sitio web con multitud de ideas para aplicar la } \\
\text { matemática con el mundo real: cuentos, imágenes, } \\
\text { sellos con inspiración matemática, canciones, usos en el } \\
\text { arte, la medicina o el deporte. También se estructura por } \\
\text { niveles educativos, perfecta para curiosear y extraer un } \\
\text { montón de } \\
\text { para la clase. }\end{array}$ \\
\hline & $\begin{array}{l}\text { Matemática } \\
\text { cine }\end{array}$ & $\begin{array}{l}\text { Blog del profesor Ángel Requena Fraile dedicado a } \\
\text { comentar y recomendar películas en las que los } \\
\text { conceptos matemáticos tienen mayor o menor } \\
\text { protagonismo. Siendo una forma de afrontar la } \\
\text { asignatura a través del séptimo arte. }\end{array}$ \\
\hline & $\begin{array}{l}\text { Experiencing } \\
\text { Maths }\end{array}$ & $\begin{array}{l}\text { Mini sitio con propuestas educativas para poner en } \\
\text { práctica la matemática observando el mundo que nos } \\
\text { rodea e interactuando proactivamente. }\end{array}$ \\
\hline
\end{tabular}

Fuente: Andrade, et. al. https://revistaespacios.com/a20v41n11/a20v41n11p07.pdf

Vista y analizada la tabla, se puede apreciar la existencia de una gran cantidad de aplicaciones educativas, ya sea en formato de páginas web en línea o en formato de recursos educativos que se orientan en función de una diversidad de categorías o subáreas de las Matemáticas de las cuáles sólo un reducido número hace una diferenciación en el aprendizaje por niveles de educación.

En este sentido, el empleo de la información y los datos al realizarse 
la clase, es decir, al trabajar con los contenidos y las representaciones en función de la información, por ejemplo la multimedia ha jugado un papel fundamental, ha revolucionado en mayor medida el tradicionalismo en la enseñanza de las matemáticas, ya que a partir de videos, y de imágenes se pueden trabajar mejor determinados contenidos.

En cuanto a esto, las presentaciones gráficas se pueden ubicar en los repositorios, en plataformas de imágenes, en páginas Web, entre otros hospedadores de estos recursos, lo que aumenta las posibilidades de acceso y lo categoriza como materiales de estudio que han sido analizados, mejorados y serán del uso de los docentes y estudiantes.

Las diversas herramientas que se pueden utilizar, favorecen la integración de información, contenidos y procedimientos, así como también permiten un mayor control en el flujo y el trabajo con imágenes, con vídeos, audios, animaciones y gráficos para ser combinados en algo que es superior a la suma de sus partes. Por lo que favorece las transiciones entre escenas, elementos para la interfaz, usuario y acceso a los datos en dispositivos de almacenamiento masivo.

Por tanto, es necesario tener presente además que el empleo de las TIC en el salón de clases para impartir la asignatura de matemática se centra en:

D Enseñan contenidos de matemática y su nivel de aplicabilidad.

Aplicaciones dirigidas a la orientación vocacional y la formación profesional, que incluyen bloques de conocimientos sobre las matemáticas y las profesionales o su nivel de aplicabilidad.

> Aplicaciones que transmiten información general (diccionario, enciclopedias, temáticas, etc.)

En lo que va de siglo XXI en general, y con mayor énfasis en los últimos años, se viene utilizando las TIC para la Enseñanza Asistida por Computadora, la rama que investiga sobre el uso de estas técnicas en el desarrollo de sistema educativo recibe el nombre de Enseñanza Inteligente Asistida por Computadora (E.I.A.C.); la cual no 
sólo aplica técnicas en los tutoriales, sino también en muchos otros tipos de entornos instructivos, en los que desatacan el desarrollo de los contenidos de las ciencias, sobre todo la matemática.

En particular, para el desarrollo de la matemática, por su amplio campo de conceptos, teoremas, y un pensamiento abstracto para su aprendizaje, a través de los diversos dispositivos electrónicos que constituyen las TIC se utilizan materiales llamados tutoriales inteligentes y las redes que guían razonablemente el proceso enseñanza-aprendizaje en particular, lo que incluso llegan a modificar el rol del docente, ya que llegan incluso a asumir las funciones de evaluación, tutor de entrenamientos, transmisor de información.

Muy a tono, se requiere tener en cuenta el espacio donde se desarrollan las diversas operaciones con los recursos digitales y demás herramientas. Es el caso del llamado espacio virtual, el cual se configura como instrumento ideal para el aprendizaje de la recuperación, o como laboratorio de experimentación ontológica. Desde esta perspectiva, Banet (2001), afirma que los espacios virtuales no son una representación de la realidad, sino, la inmersión en una realidad sintética, es decir, un espacio que se puede construir a medida que se transita, y se recorre. En tanto, se llega a la conclusión desde esa visión, que la realidad virtual es desmedida. En ella, las proporciones no son el orden en sí, sino un orden entre otros.

Es importante para el desarrollo de los contenidos de la matemática con el empleo de la virtualización, como forma de desarrollo del proceso con la mediación de las TIC en mayor medida. Por ello hay que profundizar en esos aspectos, y en tal sentido Álvarez (2002) en "La Educación Virtual" enmarca la utilización de las TIC en general, hacia el desarrollo de metodologías alternativas para el aprendizaje de alumnos de poblaciones especiales que están limitadas por su ubicación geográfica, la calidad de docencia y el tiempo disponible. Lara (2002), afirma que la Educación Virtual es "la modalidad educativa que eleva la calidad de la enseñanza aprendizaje... que respeta su flexibilidad 0 disponibilidad (en 
cualquier momento, tiempo y espacio). Alcanza su apogeo con la tecnología hasta integrar los tres métodos: asincrónica, sincrónica y autoformación".

Para la UNESCO (1998), son "entornos de aprendizajes que constituyen una forma totalmente nueva, en relación con la tecnología educativa... un programa informático - interactivo de carácter pedagógico que posee una capacidad de comunicación integrada. Son una innovación relativamente reciente y fruto de la convergencia de las tecnologías informáticas y de telecomunicaciones que se ha intensificado durante los últimos diez años".

\section{Características de educación virtual}

Al tenerse en cuenta lo planteado por Álvarez, (2002), se describe las características generales de educación virtual de la siguiente forma:

Es oportuno para datos, textos, gráficos, sonido, voz e imágenes mediante la programación periódica de tele clases.
Es eficiente, porque tiene mensajes, conferencias, etc. en forma simultánea para los centros de influencia.

> Es económico, porque no es necesario desplazarse hasta la presencia del docente o hasta el centro educativo.

> Soluciona dificultad del experto, a que viaje largos trayectos.

- Es compatible con la educación presencial en cumplimiento del programa académico.

> Es innovador según la motivación interactivo de nuevos escenarios de aprendizaje

> Es motivador en el aprendizaje, que estar inclaustrado en cuatro paredes del aula.

$>$ Es actual, porque permite conocer las últimas novedades a través de Internet y sistemas de información.

> Por otro lado, María Enriqueta Reyes, describe las dimensiones de la educación virtual:

> Ubicación relativa entre el educador - educando.

> Es instantáneo en el tiempo, pero en diferente lugar.

> El aprendizaje es a distancia, con offline o On-line en tiempo real. 
Es aprendizaje es interactivo, tanto de redes y materiales de estudio.

$>$ Es autoeducativos en ambientes multimedia 0 por módulos impresos, todos ellos centralizados en un mismo lugar, se le denomina sistemas de autoprendizaje.

- El educando no requiere concurrir al centro de estudio, pero se puede realizar trabajos y debates en comunidades virtuales.

Son posibilidades que garantizan una visión para desarrollar los contenidos de matemática desde esta perspectiva, con el empleo de las TIC y la virtualización en particular en el proceso.

En ello, al utilizar las TIC se debe tener presente en mayor medida que la enseñanza y el aprendizaje se configura con el empleo de una serie de herramientas, las cuales son decisivas y de gran utilidad ya que son:

Interactivos, los usuarios pueden adoptar un papel activo en relación al ritmo de aprendizaje.
Multimedia, ya se incorpora a textos, imágenes fijas, animaciones, videos, sonidos.

Abierta, permite una actualización de los contenidos y las actividades de forma permanente, algo que los libros de textos no poseen.

> Sincrónicos y asincrónicos, los alumnos pueden participar en las tareas y actividades en el mismo momento independientemente y en cualquier lugar (Sincrónico). O bien, la realización del trabajo y estudio individual en el tiempo particular de cada alumno (asincrónico).

Accesibles, no existen limitaciones geográficas ya que utiliza todas las potencialidades de la red de Internet, de manera que los mercados de formación son abiertas.

> Distribuidos, no tienen por qué estar centrado en un solo lugar, sino accesible en cualquier lugar del mundo, los recursos y materiales didácticos.

$>$ Con un alto seguimiento, el trabajo y actividad de los alumnos, ya que organiza tareas a tiempo a remitir.

> Comunicación horizontal, entre los alumnos, ya que la formación 
y colaboración parte de las técnicas de formación.

Características de la matemática virtual se expresan desde el protagonismo en el proceso de enseñanza aprendizaje de las TIC:

Proporciona una representación visual de los temas relacionados con la matemática que no son accesibles al estudiante de otro modo.

Le ofrece al estudiante la mejor ayuda para superar con éxito todas las dificultades en el aprendizaje y la comprensión de las matemáticas.

> Combina a la perfección el texto con numerosas ilustraciones (fotografías, dibujos, tablas y gráficos).

Los contenidos de la Matemática se exponen de manera muy pedagógica gracias a los dibujos que proporciona este curso y ello se puede relacionar con la teoría.

> Estimula la producción, reproducción, reestructuración de los contenidos.

> La Matemática virtual propone ejercicios y aplicaciones prácticas de los conocimientos adquiridos.

Combinando la información con la propuesta lúdica, las actividades de resolución de problemas y la evaluación.

$>$ Permite la actividad del estudiante.

\section{Discusión}

Se procedió al análisis de los resultados que tributan cada tarea investigativa a partir de los indicadores expresados en la operacionalización que le dan salida a los objetivos propuestos.

Para este análisis se tuvo en cuenta lo referente al empleo de las TIC en el desarrollo de los contenidos de matemática en los procesos de enseñanza aprendizaje, para lo cual se desarrolló el cuasi-experimento sobre las actividades en el proceso de enseñanza aprendizaje de la matemática con dos paralelos, el grupo de control con 31 estudiantes y el grupo experimental con un total de 32 estudiantes correspondiente a los paralelos $A$ y $B$ de educación básica superior de la unidad educativa "Camilo Ponce Enríque". 
En tal sentido se tuvieron en cuenta como principales resultados los siguientes:

Tabla 2. Diagnóstico inicial y final de la calidad del aprendizaje del grupo control.

\begin{tabular}{|c|c|c|c|c|}
\hline \multirow{2}{*}{ CATEGORÍA } & \multicolumn{2}{|c|}{ GRUPO CONTROL (antes) } & \multicolumn{2}{c|}{ GRUPO CONTROL (después) } \\
\cline { 2 - 5 } & $\mathbf{f i}$ & $\mathbf{p i}$ & $\mathbf{f i}$ & $\mathbf{P i}$ \\
\hline Excelente & 5 & 16.1 & 4 & 12.9 \\
Bueno & 8 & 25.8 & 8 & 25.8 \\
Regular & 10 & 32.2 & 10 & 32.2 \\
Malo & 8 & 25.8 & 9 & 29.0 \\
\hline & 31 & $100 \%$ & 31 & $100 \%$ \\
\hline
\end{tabular}

Fuente: Elaboración de los autores.

En el diagnóstico inicial del grupo control, el $32.2 \%$ de los estudiantes obtienen un calificativo de regular, correspondiéndole una nota de 10 puntos, se infiere que existe un escaso conocimiento de temas relacionados a la Matemática desde el empleo de las TIC en la clase y sus actividades de estudio, debido a que han aprendido esta asignatura con metodologías que no desarrollan ni fomentan la participación de los estudiantes sino al contrario simplemente recepcionan los conocimiento del profesor, y no se realizan acciones de búsqueda y profundización con las posibilidades que ofrece las TIC para ello. Seguidamente, el $25.8 \%$ de los estudiantes de este grupo obtienen un calificativo de bueno (14 puntos).
Este porcentaje representa 8 estudiantes que esencialmente respondieron preguntas relacionados con funciones básicas de la matemática en cambio no respondieron aquellas relacionadas a funciones trigonométricas, que para ello pudieron utilizar software y materiales donde podían profundizar y aprenderlo con mayor facilidad.

Un $25.8 \%$ obtuvieron el calificativo de malo (05 puntos) el cual representa 8 estudiantes del total, este grupo de estudiantes no reconocen conceptos, ni tampoco resuelven ningún tipo de ejercicios. En cambio el $16.1 \%$ que representa a 5 estudiantes de este grupo obtuvieron el calificativo de excelente (18 puntos). Ello indica 
que un menor porcentaje de estudiantes manejan la información teórica y la aplican a la resolución de problemas planteados, desde el empleo además de las TIC en las propias actividades de estudio.

Sin embrago, en el diagnóstico final del grupo de control, el calificativo que obtiene mayor porcentaje es regular (10 puntos) con un $32.2 \%$, en comparación con el diagnóstico inicial de este grupo no ha variado debido a que se desarrolló la asignatura con metodologías que no fomentaban la participación de los estudiantes ni se centraban en el empleo de multimedios, recursos tecnológicos y digitales para profundizar en los contenidos y para que fuesen didácticamente más claros en mayor medida, ni mucho menos que entre pares no compartían información.

Seguidamente, se encuentra con $25.8 \%$ los calificativos de bueno (14 puntos) y el $12.9 \%$ excelente (18 puntos), si bien es cierto que el calificativo de excelente sufre una variación aproximadamente de $4 \%$ en cambio el calificativo bueno se mantiene.

Todo lo anterior indica que este grupo de estudiante han mejorado la parte de la comprensión de información que tiene que ver con el manejo de la información teórica de los diferentes temas de matemática, en cuanto a las posibilidades para realizar las búsquedas y trabajar con los conceptos, en cambio lo referente a la profundización e experimentación no ha tenido mayor mejora debido a que con la metodología utilizada por el docente, demasiado teórica, sin emplear software y recursos digitales que pueden ser más agradables y agradables al estudiantes, que favorezcan la motivación por la matemática incluso, pues no permite la participación de los estudiantes ni mucho menos ellos pueden compartir información.

Tabla 3. Diagnóstico inicial y final de la calidad del aprendizaje del grupo experimental.

\begin{tabular}{|l|c|c|c|c|}
\hline \multirow{2}{*}{ CATEGORÍA } & \multicolumn{2}{|c|}{ GRUPO EXPERIMENTAL (antes) } & \multicolumn{2}{c|}{ GRUPO EXPERIMENTAL (después) } \\
\cline { 2 - 5 } & $\mathbf{f i}$ & $\mathbf{P i}$ & $\mathbf{f i}$ & $\mathbf{P i}$ \\
\hline Excelente & 6 & 19.0 & 18 & 56.0 \\
Bueno & 7 & 22.0 & 14 & 44.0 \\
\hline
\end{tabular}




\begin{tabular}{|l|c|c|c|c|}
\hline Regular & 11 & 34.0 & 0 & 00.0 \\
Malo & 8 & 25.0 & 0 & 00.0 \\
\hline & 32 & $100 \%$ & 32 & $100 \%$ \\
\hline
\end{tabular}

Fuente: Elaboración de los autores.

En el diagnóstico inicial del grupo experimental y de acuerdo a las respuestas emitidas por los estudiantes de este grupo se tiene un conocimiento regular de los principales contenidos tratados en la asignatura de matemática, ya que representa el $34 \%$ de los estudiantes. De este grupo el $19 \%$ que son 6 estudiantes obtuvieron el calificativo de excelente y el $22 \%$ tienen un calificativo bueno. Se infiere que los estudiantes tienen conocimientos muy pobres respecto a esta asignatura, y no poseen además conocimientos abundantes en el desarrollo de estrategias de aprendizaje y de estudio con el empleo de las TIC, en la cual la explicación podría deducirse que ellos, también han sido formados con metodologías tradicionales que no favorece la actividad de ellos mucho menos participar en el desarrollo de las actividades de aprendizaje.

En el diagnóstico final del grupo experimental el $56 \%$ obtuvo el calificativo de excelente (18 puntos), en comparación con el diagnóstico inicial se observa que el aumento es significativo. Lo que indica que cuando los estudiantes al estar presentes con una metodología abundante en el empleo de las TIC por parte de los docentes, y en ellos mismos en su manipulación y desarrollo de actividades desde esta perspectiva. Por ello, se constata que los estudiantes participan de manera individual o grupal en la adquisición de información y luego lo aplican, al mismo tiempo comparten opiniones de forma sincrónica 0 asíncrona, en foros $u$ otras alternativas, mediante un debate que favorecen y mejoran sus aprendizajes paulatinamente. De la misma manera realizan la indagación e experimentación mediante la resolución de ejercicios con el empleo de ciertos software que el docente introdujo en sus clases y que los enseñó a utilizar, debido a que el $44 \%$ de los estudiantes tienen el calificativo de bueno (14 puntos), al realizar una comparación con el diagnóstico 
inicial se evidencia con este calificativo al $22 \%$. De lo cual se infiere que cuando los estudiantes son protagonistas de su propia formación al participar y estar más motivados, ya que usan herramientas que le son más adecuadas a sus posibilidades, como mediación para sus aprendizajes y alternativas de desempeño en el desarrollo de dicho aprendizaje. Por lo que participan con mayor regularidad en los debates, ofrecen en este sentido aportes, ideas u opiniones, las que se refuerzan con las imágenes que presenta la multimedia en el cual ellos (as) pueden apreciar y reconocer los procesos de resolución de los ejercicios, y no solo eso; sino también pueden relacionarlos con sus funciones respectivas.

Llama la atención el hecho que ningún estudiante obtiene el calificativo de regular y malo. A partir de ello y al observar el diagnóstico inicial, se infiere que a medida que se aplica el sistema virtual y el empleo de las TIC en el aprendizaje de las matemáticas los estudiantes mejoran sus aprendizajes. Ya que ellos participan en este proceso, incluso aquellos estudiantes que anteriormente tenían dificultades en el aprendizaje superan lo dicho porque el ambiente social y el sistema virtual obliga a que participen activamente conjuntamente con sus compañeros ello demuestra los resultados.

\section{Conclusiones}

En las instituciones de educación básica superior, el uso de plataformas virtuales en lugar de herramientas de apoyo a la formación profesional debe estar sesgado hacia el proceso docente, como propósito fundamental, incluso debe estar vinculado a la docencia en todo momento para mejoras en el aprendizaje de los estudiantes. Las innovaciones en el empleo de las TIC han fortalecido la relación en los componentes personales se incluso no personales del proceso de enseñanza aprendizaje, y han hecho efectivos los resultados del aprendizaje. La sociedad inmersa en el mundo tecnológico requiere de un espacio de interacción digital para convertirse en un espacio de innovación educativa. Además, también es muy importante 
reconocer

las

acciones

implementadas a través de las diversas herramientas, recursos y plataformas para ello, e incluso las características de las acciones que se consideran innovadoras.

El proceso con un enfoque desde la virtualidad o simplemente el empleo de las TIC mejora el aprendizajes de la Matemáticas debido a que los alumnos observan y comprenden los contenidos a su vez desarrollan habilidades y destrezas.

En términos generales, el uso de plataformas virtuales y la formación con apoyo pedagógico y técnico son cruciales para los docentes y sus desempeños que se reflejan en los aprendizajes de los estudiantes. Una buena evaluación de la tecnología en la docencia debe aprovechar las ventajas que se han logrado en la práctica innovadora. En ese sentido, se puede concluir en que el uso de las TIC como estrategia didáctica en el aprendizaje de las matemáticas de los estudiantes de educación general básica superior favorece significativamente el proceso de enseñanza y aprendizaje.

\section{Bibliografía}

Álvarez, O. H., \& Zapata, D. Z. (2002). La enseñanza virtual en la educación superior. Icfes.

Andrade, C. de los Ángeles, M., Jaramillo, L. Leonardo, M., Caraguay, M. Cecibel, G., ... \& Armando, W. Las Tics como herramienta metodológica en matemática.

Banet, M. (2001). Paradoja de los Entornos virtuales. México: Editorial April.

Cadena-Vela, J. Ortiz Herrera, G. Torres and G. Mejía-Madrid. (2018). Innovation in the university: virtual educational platform. In Proceedings of the 6th International Conference on Technological Ecosystems for Enhancing Multiculturality (TEEM 2018)

Cerrillo-i-Martínez, A. (2012). La contribución de las TIC a la mejora de la transparencia administrativa. Arbor, 188(756), 707-724.

Chigozie-Okwum, Chioma. (2016). INNOVATIVE PEDAGOGIES IN EDUCATIONAL TECHNOLOGY; THE CLOUD COMPUTING EXPERIENCE. Ideal Journal of Education and Policy Studies. 2. 192-200.

Davis, J. P., \& Bellocchi, A. (2020). Gamification of SSI's as a science pedagogy: Toward a 
critical rationality in teaching science. In Science Teacher Education for Responsible Citizenship (pp. 101-116). Springer, Cham.

Fahriddinqizi, Ashurova. (2020). Fundamentals of implementation of innovative educational technologies. ACADEMICIA: An International Multidisciplinary Research Journal. 10. 888890. $10.5958 / 2249-$ 7137.2020.01826.1.

García-Alcaraz, P., Martínez-Loya, V., García-Alcaraz, J. L., \& Sánchez-Ramírez, C. (2018). The Role of ICT in Educational Innovation. Managing Innovation in Highly Restrictive Environments, 143-165. doi:10.1007/978-3319-93716-8_7

Lara, L. R. (2002). Análisis de los recursos interactivos en las aulas virtuales. SegundoCongreso Virtual" Integración sin Barreras en el Siglo XXI".

Naifeld, Edni \& Simon, Eitan. (2017). Teaching

Students'Understanding of Innovative Pedagogy. European Scientific Journal. 13.

10.19044/esj.2017.v13n4p15

Pabón-Gómez, J. A. (2014). Las TICs y la lúdica como herramientas facilitadoras en el aprendizaje de la matemática. Eco matemático, 5(1), 37-48.

Parra-González, M. E., López Belmonte, J., Segura-Robles, A., \& Fuentes Cabrera, A. (2020). Active and emerging methodologies for ubiquitous education: Potentials of flipped learning and gamification. Sustainability, 12(2), 602.

Riveros, V., Bernal, M. I. M., \& Castro, R. (2011). Las tecnologías de la información y la comunicación en el proceso de instrucción de la matemática.

Quórum académico, 8(15), 111-130.

Rossi Cordero, Barajas Frutos, (2018). Digital competence and educational innovation: challenges and opportunities. Profesorado on September 01, 2018

Siakas, Kerstin \& Rahanu, Harjinder \& Georgiadou, Elli \& Paltalidis, Nickos. (2019). Education and Social Development Current Pedagogical Trends. DEStech Transactions on Social Science, Education and Human Science. 10.12783/dtssehs/icesd2019/ 28060 .

Silva, D. de A. (2018). Using Innovative Pedagogy to Connect School and 
Community for Democratic Impact. The Educational Forum, 82(3), 374-377. doi:10.1080/00131725.2018. 1457120

Singh, Jaswinder. (2020). Encouragement at the HEART of Education Innovation. Childhood Education. $\quad 96$. 60-65. 10.1080/00094056.2020.179 6457.

Sousa, A. T. O. de, Formiga, N. S., Oliveira, S. H. dos S., Costa, M. M. L., \& Soares, M. J. G. O. (2015). A utilização da teoria da aprendizagem significativa no ensino da Enfermagem. Revista Brasileira de Enfermagem, 68(4), 713-722. doi:10.1590/00347167.2015680420i

Stachová, K., Papula, J., Stacho, Z., \& Kohnová, L. (2019). External Partnerships in Employee Education and Development as the Key to Facing Industry 4.0 Challenges. Sustainability, 11(2), 345. https://doi.org/10.3390/su110 20345

Tarando, Elena \& Malinina, Tatiana \& Mishalchenko, Yuri \& Galchenko, Natalya \& Finogenko, Elena \& Syvorotkina, Irina. (2021). A new definition of the concept of "innovative education. Laplage em Revista. 7. 170-
181. 10.24115/S24466220202171684p.170-181.

Terziev, Venelin. (2020). Science as a tool for social development.. 5. 117-134. 10.19275/RSEP091.

Tippa, Naveenkumar \& Mane, Sangeetha. (2018). SOCIAL WORK EDUCATION AND SOCIAL DEVELOPMENT: REFLECTING ON FIELD WORK TRAINING. 545-549.

Tso, A. W. B., \& Lau, J. M. Y. (2018). An explorative study on the pedagogical potential of gamification. In Innovations in open and flexible education (pp. 143-151). Springer, Singapore.

UNESCO (1998a): Declaración mundial sobre la educación superior en el siglo XXI: visión y acción. UNESCO.

Utomo, A. Y., \& Santoso, H. B. (2015). Development of gamification-enriched pedagogical agent for eLearning system based on community of inquiry.

Proceedings of the International $\mathrm{HCl}$ and UX Conference in Indonesia on - CHluXiD '15. doi:10.1145/2742032.274203 3

Velázquez, M., Báez, A., Pérez, A. \& Luna, A. (2021). Educational innovation in the comprehensive training of 
nursing graduates.

International journal of health sciences.

5.

10.29332/ijhs.v5n1.700.

Warford, M. K. (2017). Educational Innovation Diffusion:

Confronting Complexities.

Reforms and Innovation in

Education,

11-36.

doi:10.1007/978-3-319-

60246-2_2 